\title{
Do Robotic and Non-Robotic Arm Movement Training Drive Motor Recovery after Stroke by a Common Neural Mechanism? Experimental Evidence and a Computational Model
}

\author{
David J. Reinkensmeyer, Member, IEEE, Marc A. Maier, Emmanuel Guigon, Vicky Chan, O. Mine \\ Akoner, Eric T. Wolbrecht, Steven C. Cramer, James E. Bobrow
}

\begin{abstract}
Different dose-matched, upper extremity rehabilitation training techniques, including robotic and nonrobotic techniques, can result in similar improvement in movement ability after stroke, suggesting they may elicit a common drive for recovery. Here we report experimental results that support the hypothesis of a common drive, and develop a computational model of a putative neural mechanism for the common drive. We compared weekly motor control recovery during robotic and unassisted movement training techniques after chronic stroke $(n=27)$, as assessed with quantitative measures of strength, speed, and coordination. The results showed that recovery in both groups followed an exponential time course with a time constant of about 4-5 weeks. Despite the greater range and speed of movement practiced by the robot group, motor recovery was very similar between the groups. The premise of the computational model is that improvements in motor control are caused by improvements in the ability to activate spared portions of the damaged corticospinal system, as learned by a biologically plausible search algorithm. Robot-assisted and unassisted training would in theory equally drive this search process.
\end{abstract}

\section{INTRODUCTION}

A common finding in upper extremity stroke rehabilitation research is that disparate but intensitymatched movement training techniques elicit similar improvements in movement ability following stroke [1-3]. A simple explanation is that different training techniques elicit a common neural drive for plasticity. However, what this common drive might be is not well understood or modeled.

Interpretation of the finding of comparable recovery with disparate training techniques is difficult because most previous clinical studies have used coarse clinical outcome measures that were assessed only a few times per patient. A patient could improve his or her score on a coarse outcome measure by changing movement control processes in several different ways with several different time courses. Quantitative details of motor control assessed frequently are

This work was supported in part by NIH N01-HD-3-3352 from NCMRR and NIBIB and by NCRR M01RR00827.

D.J.R., V.C., M.O., and JEB are with the Department of Mechanical and Aerospace Engineering, Univ of California, Irvine, 92697.

M.A. M. is with LNRS (UMR 7060 CNRS, Université Paris Descartes) 75006 Paris, France

E.G. is with ISIR (UMR 7222 CNRS, UPMC), 75005 Paris, France

E.T.W. is with the Department of Mechanical Engineering, University of Idaho

S.C.C. is with the Department of Neurology, University of California at Irvine, 92697-3975

978-1-4244-3296-7/09/\$25.00 @2009 IEEE needed to distinguish detailed patterns of recovery.

The experimental study reported here addressed these issues by comparing two movement training techniques robot-assisted and non-assisted arm movement training using quantitative measures taken at weekly intervals. The two techniques were dose-matched, but the robot-assisted participants practiced substantially different movements, in terms of range of motion (ROM) and speed, because of the robot assistance.

We found that the patterns of recovery assessed with the quantitative measures were strongly correlated between the training techniques. To explain this result, we propose a neuro-computational model of practice-dependent movement plasticity. The premise of this model is that improvements in motor function are caused by improvements in the ability to activate spared portions of the corticospinal system, which are in turn driven by trial-anderror practice and a scalar teaching signal resulting from each movement.

\section{EXPERIMENTAL METHODS}

\section{A. Subjects}

27 adult stroke survivors were recruited through local hospitals and stroke support groups in Orange County, CA. All participants had experienced a single ischemic or hemorrhagic stroke and were at least three months post stroke prior to their enrollment into the program (mean $=67$ months). All participants demonstrated moderate to severe weakness of their affected upper extremities defined by the Fugl-Meyer Motor Scale (robot group, $n=13$, mean $=25$ out of 66 , range 10 to 39 ; control group, $n=14$, mean $=23$ out of 66, range 12 to 33). Exclusion criteria included significant pain, instability or subluxation of the affected shoulder, severe elbow or wrist contractures, concurrent severe medical problems, cognitive dysfunction, visual deficits, severe neglect or apraxia, and current enrollment in ongoing upper extremity therapy. All subjects provided written consent. The study was approved by the IRB of UCI.

\section{B. Device: Pneu-Wrex}

The robotic device used ("Pneu-Wrex" [4-6]) is a four degree-of-freedom robot. It is based on a passive arm support called WREX [7], allows a wide range of motion of the arm in a 3D space, and incorporates pneumatic actuators to generate active forces. It uses an adaptive controller that 
learns the dynamics of the patient's arm, ability and effort at the same time, using an adaptive sliding controller with the novel addition of a forgetting term (see [4] for detailed description). With this controller, the device provides compliant assistance-as-needed that allows the patient to actively participate in and complete virtual tasks. Hand movement training through grasp and release is incorporated through a grip sensor. Position sensors located on the joints along the Pneu-Wrex exoskeleton allow it to be used as an input device to play computer games that are functionally oriented. Games in this study included grocery shopping, cleaning a window, playing basketball, and driving a car, and included auditory and visual feedback.

\section{Assignment and Intervention}

We compared the arm movement of subjects who participated in Pneu-Wrex training with control subjects who exercised for the same duration without the device and received similar amounts of supervision from a therapist. All subjects participated in 24 one-hour, therapistsupervised treatment sessions, approximately three times per week for eight to nine weeks. Subjects were randomly assigned to the Pneu-Wrex or control group using a block allocation method. Subjects assigned to the control group participated in conventional table-top exercises developed at the Rehabilitation Institute of Chicago [8]. These exercises included bimanual ROM stretches, active ROM exercises with tabletop support, and a list of ADL tasks.

\section{Assessment Procedures}

In this paper, we focus on the weekly quantitative assessments performed on participants in both groups with their arms in the robotic device, although other clinical assessments were also taken. These assessments included a measure of the strength of the subject's shoulder flexion and elbow extension. The endpoint of the robot was attached to a spring scale, and subjects raised their arm against the spring, or extended their arm outward from their body against the spring. Readings from the spring gauge were recorded. The speed assessment measured how fast subjects were able to move their affected arm first in an upward direction then in a horizontal direction away from their body. The coordination assessment measured the time to complete the basketball and window washing robotic training games. The basketball game required that the participant grab a ball represented on the computer screen, lift the ball, and dunk it in a hoop. The window washing game required sweeping the arm across a virtual window in the frontal plane to clean it.

\section{RESULTS}

For both the robot-trained and table-top trained groups, the weekly measures of movement ability improved significantly and exponentially over the ten week period, with a time constant of about 4-5 weeks (Figures 1 and 2). The recovery dynamics were highly correlated between the two groups for all outcome measures; when the recovery curves were regressed against each other, $\mathrm{r}^{2}$ ranged from 0.67 to $0.91, \mathrm{p}<0.005$.
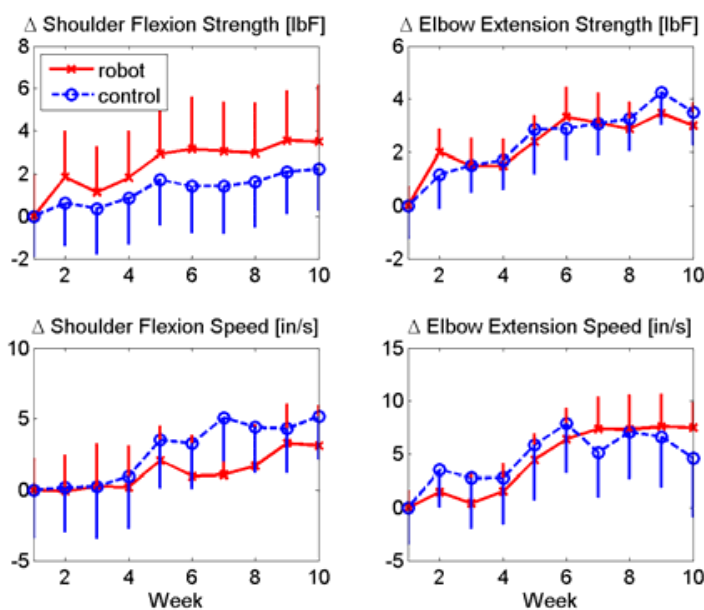

Figure 1. Change in shoulder flexion and elbow extension strength and speed over 10 weeks of robot-assisted (x) or conventional table-top (o) training. The bars show the standard error of the mean. All changes are significant comparing first to last week $(\mathrm{p}<0.05)$.
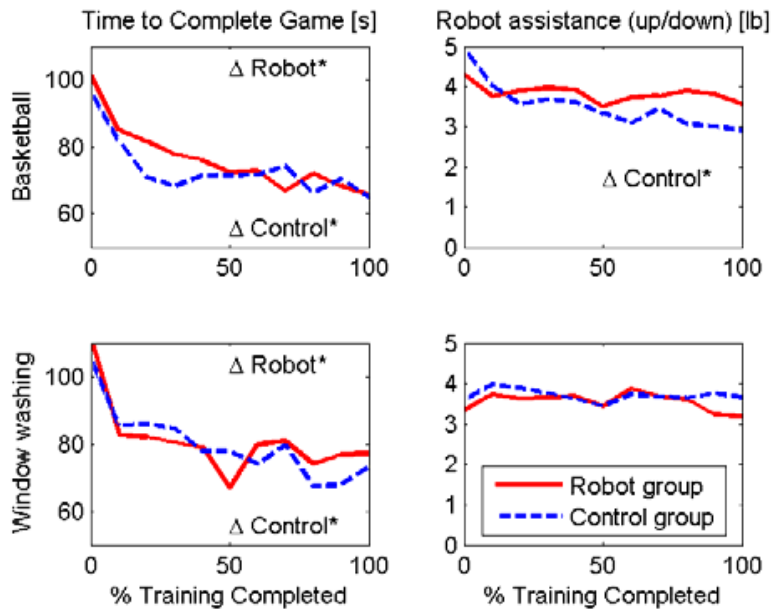

Figure 2: Left: Time to complete the basketball and window washing games for the robot (solid line) and control (dashed line) groups. Right: Amount of robot assistance in the vertical direction during game play for each game. Significant changes from start to end at $\mathrm{p}<0.05$ are labeled.

\section{DiscuSSION OF EXPERIMENTAL RESUltS}

The robot and control training techniques differed in at least three key ways. First, the participants who received assistance from the robotic exoskeleton were able to move their arms through a much wider range of movement with higher movement speeds because of the robotic assistance, as we have shown previously [4]. Second, they received a graded amount of assistance based on their movement ability, via the use of an adaptive assistance algorithm, also shown previously [4], compared to the fixed assistance level provided by the table for the control group. Third, they received quantitative feedback about their movements on the computer screen, while the control group merely had visual feedback regarding their arms. Yet the robot and control groups recovered with strikingly similar temporal dynamics. 
A simple interpretation of this finding is that the action of trying to move accounted for the practice-dependent arm movement recovery, independently of the specific ROM achieved or the level of assistance. In this interpretation, it is then the action of trying to move that automatically and inherently elicited the teaching signal required for the neural reorganization supporting the movement recovery, independently of the quantitative task feedback. In the next section, we develop a computational model of usedependent brain organization based on this interpretation.

\section{Neuro-COMPutational Model}

\section{A. Overview of the Stochastic Local Search Model}

The model focuses on strokes that partially destroy the corticospinal system, either by damaging cortical motor areas of white matter projections to the motor neuron pools from cortical motor areas. We assume that motor control gains are caused by improvements in the ability to activate the spared portions of the corticospinal tract that activate motor neuron pools. We further assume that the motor system learns how to better activate spared corticospinal tracts by searching for optimal activation patterns using a stochastic local search process [9-12]. The teaching signal that guides this search is a scalar measure of the movement success caused by the current activation pattern, relative to the most successful, previously-tried activation pattern.

\section{B. Model Details}

The model assumes that the force a muscle generates is determined by the weighted, summed activity of corticospinal (CS) cells that drive the arm muscle motor neurons pool (Fig. 3), where CS activity is defined by a vector of mean firing rates. In the simulations reported here, we modeled connectivity based on cortico-motoneuronal and other last-order pre-motor neurons, which have been well characterized using spike-triggered averaging techniques in primates [13-17]. These connections are primarily focal and excitatory. Changing the percentage of reciprocal or inhibitory connections did not alter the model findings.

We assumed that increases in the firing rate of a single CS cell caused proportional increases in muscle force [13, 18, 19], up to a saturation limit, with the proportionality constant determined by a fixed, connection weight. We experimented with versions of the model in which the saturation limits for each cell were drawn from different random distributions; the results from such models did not differ from a model in which the saturation limits were all set at the same constant, so, for simplicity, we present only a model in which the saturation limit of all excitatory cells was set to 1 and inhibitory cells to -1 .

We studied two stochastic local search algorithms that have been previously proposed as biologically plausible: a best-first stochastic search [9] and a stochastic gradient descent algorithm [11]. For the simulations reported here, we assumed the teaching signal was related to the peak force generated by an activation attempt. Specifically, the motor system searched for activation patterns that produced more force in a desired direction (flexion or extension). Thus, for the best-first search, given an initial activation pattern $\mathrm{X}_{0}$ that was measured to have produced a force $\mathrm{F}_{0}$ for the target motor function, the motor system tries a new pattern $\mathrm{X}_{\mathrm{i}}=\mathrm{X}_{0}$ $+N_{i}$, where $N_{i}$ is random noise, and then measures the movement success (i.e. force) $F_{i}$ produced by this pattern. Here, the noise is assumed to arise from independent stochastic processes operating on single neurons, and is drawn from a zero-mean normal distribution with standard deviation $\sigma$, although other noise distributions (e.g. signal dependent) are possible. Then, plasticity occurs if $F_{i}>F_{0}$, in which case $\mathrm{X}_{0}=\mathrm{X}_{\mathrm{i}}$ and $\mathrm{F}_{0}=\mathrm{F}_{\mathrm{i}}$. That is, the motor system memorizes the new pattern $X_{i}$ if the force $F_{i}$ it produces is greater than the force $F_{0}$ of the best previously tried movement. The memorization of the new pattern $X_{i}$ is assumed to occur by trigger of a neuromodulatory signal that controls synaptic weights $\mathrm{w}_{\mathrm{i}}$ between a command signal $U_{i}$ and the corticospinal cell activation vector $X_{i}$ (Fig. 3).

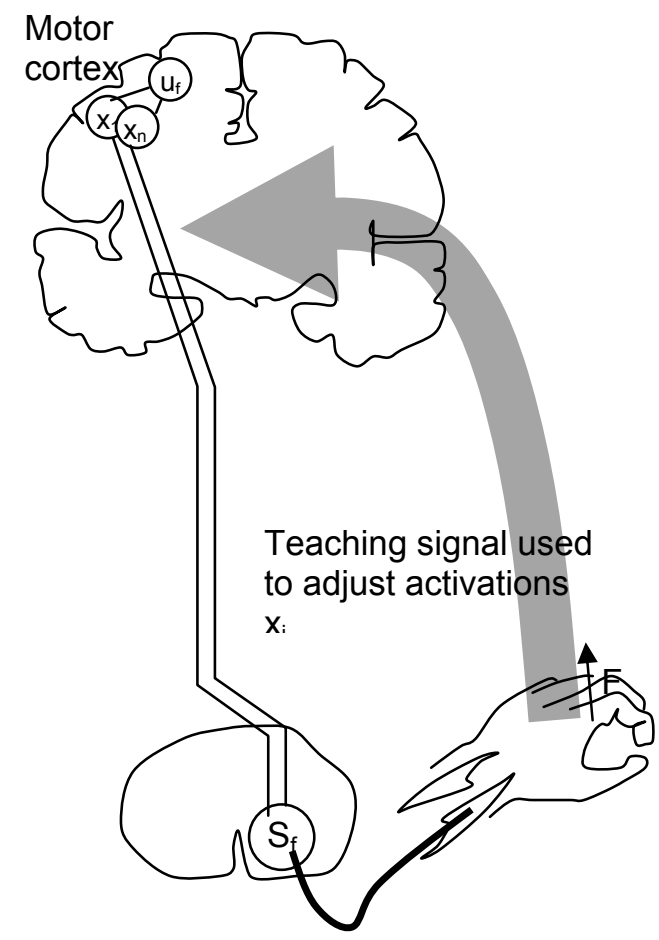

Figure 3. Stochastic search model of motor control recovery. A. The model focuses on how the brain learns to adjust the activations $\mathrm{x}_{\mathrm{i}}$ of $\mathrm{N}$ residual corticospinal (CS) cells in response to a motor command $\mathrm{u}_{\mathrm{f}}$ to create a drive $\mathrm{S}_{\mathrm{f}}$ to motor neuron pools and create a force $\mathrm{F}$.

\section{Model Results}

The stochastic local search mechanism optimized the activities of the simulated CS network, increasing the force output of the simulated arm with movement practice (Fig. 4). The search mechanism causes an exponential-like force recovery curve, with a slow increase late in practice rather than an asymptote, similar to the experimentally measured strength, speed, and coordination recovery curves (cf. Fig.4 
with Figs. 1 and 2). The range and speed of the practiced movements do not alter the rate of recovery; all that matters is the magnitude of the teaching signal (in this case peak force) achieved by the current pattern of activation.
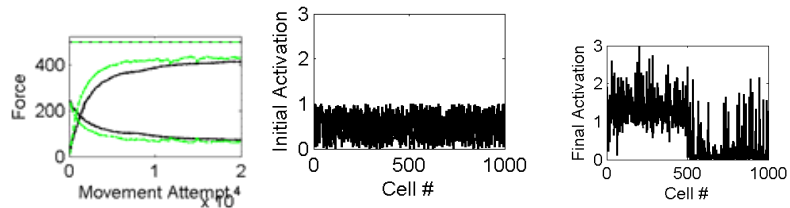

Figure 4. Strength recovery and activation optimization by stochastic search. Left: Example force recovery curves using best-first search (black line) and stochastic gradient descent (green). The decreasing curves show the antagonist force, which equals the amount of co-contraction. The network had 500 agonist-facilitating and 500 antagonist-facilitating CS cells, the peak force possible from an individual unit was 1.0, and the noise was zero-mean Gaussian with $\sigma=0.02$. The learning gain $g$ for stochastic gradient descent was 0.8 . The maximum force possible from the CS network was 500 (dotted line). Middle: Initial cell activity was randomized with a uniform distribution from 0 to 1 . Right: Cell activity after 20,000 movement attempts. The agonist-facilitating cells' activity increased.

\section{DISCUSSION}

The experimental evidence reported here suggests that practicing movements with or without robotic assistance produces a similar time-course and magnitude of motor recovery after chronic stroke. A simple interpretation of this finding is that the action of trying to move, along with a scalar teaching signal about movement success, drives practice-dependent arm movement recovery, independently of the level of assistance. We presented a model of motor plasticity consistent with this interpretation, in which the brain searches for better ways to activate motor neuron pools by trying new patterns that are slightly and randomly perturbed from previous patterns. Such a model predicts exponential-like recovery driven by practice regardless of range or speed of the practiced movement. In other words, the amount of robot assistance does not alter recovery.

Additional simulations with this model indicate that it is competent to make other predictions consistent with clinical and physiological data, including that:

- Early strength will predict late strength recovery

- The ultimate strength attainable will be proportional to the number of spared corticospinal fibers

- There will exist a residual capacity for further strength increases with further practice

- The differential effect of a dose of movement practice will be greater earlier in recovery

- Force-related brain activation will increase in secondary motor areas following stroke, where a secondary motor area is defined as one with weaker drive to motor neuron pools

The model also makes interesting novel predictions:

- Temporarily inhibiting subpopulations of more powerfully connected corticospinal neurons will allow the motor system to optimize descending systems with a weaker influence.

- Randomly varying the dynamic environment experienced during training will slow learning by making it impossible for the motor system to effectively compare the results of the current motor pattern with previous "best" patterns.

Future work will test these predictions.

\section{REFERENCES}

[1] L. E. Kahn, M. L. Zygman, W. Z. Rymer and D. J. Reinkensmeyer, "Robot-assisted reaching exercise promotes arm movement recovery in chronic hemiparetic stroke: A randomized controlled pilot study," Journal of Neuroengineering and Neurorehabilitation, vol. 3:12, 2006.

[2] G. Kwakkel, B. J. Kollen and H. I. Krebs, "Effects of robot-assisted therapy on upper limb recovery after stroke: a systematic review," Neurorehabil. Neural Repair, vol. 22, pp. 111-121, Mar-Apr. 2008.

[3] B. T. Volpe, D. Lynch, A. Rykman-Berland, M. Ferraro, M. Galgano, N. Hogan and H. I. Krebs, "Intensive sensorimotor arm training mediated by therapist or robot improves hemiparesis in patients with chronic stroke," Neurorehabil. Neural Repair, vol. 22, pp. 305-310, May-Jun. 2008.

[4] E. T. Wolbrecht, C. V, D. J. Reinkensmeyer and J. E. Bobrow, "Optimizing compliant, model-based robotic assistance to promote neurorehabilitation," IEEE Transactions Neural Systems and Rehabiltation Engineering, vol. 16, pp. 286-297, 2008.

[5] E. T. Wolbrecht, D. J. Reinkensmeyer and J. E. Bobrow, "Pneumatic Control of Robots for Rehabilitation," Submitted to the International Journal of Robotics Research, 2008.

[6] R. J. Sanchez, E. Wolbrecht, R. Smith, J. Liu, S. Rao, S. Cramer, T. Rahman, J. E. Bobrow and D. J. Reinkensmeyer, "A pneumatic robot for retraining arm movement after stroke: Rationale and mechanical design," Rehabilitation Robotics, 2005. ICORR 2005. 9th International Conference on, pp. 500-504, 2005.

[7] T. Rahman, W. Sample, R. Seliktar, M. Alexander and M. Scavina, "A body-powered functional upper limb orthosis," Journal of Rehabilitation Research and Development, vol. 37, pp. 675-80, 2000.

[8] S. J. Housman, K. M. Scott and D. J. Reinkensmeyer, "A Randomized Controlled Trial of Gravity-Supported, Computer-Enhanced Arm Exercise for Individuals With Severe Hemiparesis," Neurorehabilitation and Neural Repair, Epub ahead of print, 2009.

[9] R. W. Anderson, "Random-walk learning: A neurobiological correlate to trial-and-error," in Progress in Neural Networks O. M. Omidvar and J. Dayhoff, Eds. Boston: Academic Press, 1988, pp. 221-244.

[10] R. J. Williams, "Simple statistical gradient following algorithms for connectionist reinforcement learning," Mach Learn, 8:229-256, 1992.

[11] J. Werfel, X. Xie and H. S. Seung, "Learning curves for stochastic gradient descent in linear feedforward networks," Neural Comput., vol. 17, pp. 2699-2718, Dec. 2005.

[12] P. Mazzoni, R. A. Andersen and M. I. Jordan, "A more biologically plausible learning rule for neural networks," Proc. Natl. Acad. Sci. U. S. A., vol. 88, pp. 4433-4437, May 15. 1991.

[13] M. A. Maier, S. I. Perlmutter and E. E. Fetz, "Response patterns and force relations of monkey spinal interneurons during active wrist movement," J. Neurophysiol., vol. 80, pp. 2495-2513, Nov. 1998.

[14] S. I. Perlmutter, M. A. Maier and E. E. Fetz, "Activity of spinal interneurons and their effects on forearm muscles during voluntary wrist movements in the monkey," J. Neurophys., vol. 80, pp. 2475-2494, 1998.

[15] E. E. Fetz and P. D. Cheney, "Postspike facilitation of forelimb muscle activity by primate corticomotoneuronal cells," J. Neurophysiol., vol. 44, pp. 751-772, Oct. 1980.

[16] R. J. Kasser and P. D. Cheney, "Characteristics of corticomotoneuronal postspike facilitation and reciprocal suppression of EMG activity in the monkey," J. Neurophy., vol. 53, pp. 959-978, 1985.

[17] K. Mewes and P. D. Cheney, "Facilitation and suppression of wrist and digit muscles from single rubromotoneuronal cells in the awake monkey," $J$. Neurophysiol., vol. 66, pp. 1965-1977, Dec. 1991.

[18] E. E. Fetz, P. D. Cheney, K. Mewes and S. Palmer, "Control of forelimb muscle activity by populations of corticomotoneuronal and rubromotoneuronal cells," Prog in Brain Res, vol. 80, pp. 437-449, 1989.

[19] J. Ashe, "Force and the motor cortex." Behavioural Brain Research, vol. 87, pp. 255-69, 1997. 